\title{
The Use of Response Surface Methodology to Evaluate the Impact of Level 2 SUPAC-IR Changes on the In Vitro Release of Metronidazole and Ranitidine from a Fixed-Dose Combination Tablet
}

Loti D. King'ori and Roderick B. Walker*

Division of Pharmaceutics, Faculty of Pharmacy, Rhodes University, P.O. Box 94, Grahamstown 6140, South Africa

\begin{abstract}
The purpose of this study was to evaluate the effect of different levels of disintegrant (croscarmellose sodium, CCS), binder (polyvinylprrolidone K30, PVP-K30), and lubricant (magnesium stearate) on the in vitro release of metronidazole (MTZ) and rantidine (RTD) from a solid oral fixed-dose combination tablet. The excipient levels investigated were Level 2 changes in component and composition described in the Scale-Up and Post Approval Changes for Immediate Release (SUPAC-IR) guidance (1). Batches of tablets (1000 units) were manufactured by wet granulation using a Saral high-shear mixer granulator and a Manesty B3B rotary tablet press. Weight uniformity, friability, and disintegration of all tablets were assessed, and all batches complied with compendial specifications. The amount of drug released $(Q)$ at ten minutes was dependent on the levels of CCS in the formulation, and the effect of PVP-K30 and magnesium stearate was dependent on the levels of CCS. Synergistic interactions between independent variables were observed for the $Q_{10}$ value for RTD, whereas PVP-K30 and magnesium stearate exhibited an antagonistic effect on the $Q_{10}$ values for MTZ and RTD. The use of response surface methodology facilitated an investigation into the effect of Level 2 component and composition changes, as described in SUPAC-IR, on the in vitro release of MTZ and RTD from a fixed-dose combination (FDC) solid oral dosage form (SODF).
\end{abstract}

\section{INTRODUCTION}

S tatistical design of experiments (DOE) has been used in the pharmaceutical industry to facilitate the optimization of manufacturing processes, formulations, and analytical methods (2) for over three decades $(3,4)$. The use of DOE permits the formulation scientist to study simultaneously the effects of multiple factors that may impact or determine product quality during the optimization process $(5,6)$. Furthermore, mathematical models can be generated to produce graphical representations that describe the variability of responses from a system as a function of the predetermined input factors thought to impact the manufacturing procedure (5). The application of statistical design and mathematical equations in the development, improvement, or optimization of pharmaceutical processes is defined as response surface methodology (RSM) (6-9).

The use of RSM for the determination of significant input factors in the in vitro release of an active pharmaceutical ingredient (API) from dosage forms is a common phenomenon (10-12). However, in most cases, RSM has been used to establish the effect of input factors on the in vitro release of solid dosage forms that contain a single API $(13,14)$. Consequently, this study aimed to investigate the effect of Level 2 component or composition changes

${ }^{*}$ Corresponding author. described in SUPAC-IR on the in vitro release of a SODF that comprised two API in a fixed-dose combination.

MTZ and RTD are Class 1 and 3 compounds, respectively, as defined by the Biopharmaceutics Classification System (BCS) (15) and are both used for the treatment of peptic or duodenal ulcers (16). MTZ and RTD, both highly water-soluble, were selected to investigate the effect(s) of different levels of disintegrant, binder, and lubricant on the in vitro release profiles of these compounds from FDC tablets.

\section{METHODS}

\section{Materials}

RTD and MTZ were purchased from Changzhou Longcheng Medicine Raw Material Co., Ltd, (Changzhou City, Jiangsu, China) and Huanggang Hongya Pharmaceutical Co. Ltd (Huanggang, Hubei, China), respectively. Analytical standards for HPLC analyses were procured from Sigma Aldrich (St Louis, Missouri, USA). Microcrystalline cellulose PH 102 (MCC), polyvinylpyrrolidone-K30 (PVP-K30), croscarmellose sodium (CCS), colloidal silicon dioxide, and magnesium stearate were purchased from Aspen Pharmacare (Port Elizabeth, Eastern Cape, South Africa). Acetonitrile (200-nm UV cutoff) and methanol (215-nm UV cutoff) were purchased from Romil Ltd (Waterbeach, Cambridge, UK). Sodium hydroxide pellets and potassium dihydrogen orthophosphate were purchased from Merck Chemicals Ltd (Modderfontein, 
Table 1. Formulation Used for the Manufacture of MTZ and RTD Tablets

\begin{tabular}{|c|c|c|}
\hline \multirow[b]{2}{*}{ Raw material } & \multicolumn{2}{|c|}{ Composition } \\
\hline & $(\% \mathrm{w} / w)$ & Actual Amount (g) \\
\hline Ranitidine & 16.8 & 84.0 \\
\hline Metronidazole & 50.0 & 250.0 \\
\hline Microcrystalline cellulose & 27.0 & 135.0 \\
\hline Polyvinyl pyrrolidone-K30 & 1.0 & 5.0 \\
\hline Croscarmellose sdoium & 4.0 & 20.0 \\
\hline Colloidal silicone dioxide & 0.2 & 1.0 \\
\hline Magnesium stearate & 1.0 & 5.0 \\
\hline
\end{tabular}

Gauteng, South Africa), and triethylamine was procured from SaarChem Pty Ltd (Krugersdorp, Gauteng, South Africa).

\section{Equipment}

All raw materials were weighed using a top-loading analytical balance Model PM4600 (Mettler Instruments, Zurich, Switzerland) with a sensitivity of $0.01 \mathrm{~g}$. Blending and granulation were undertaken using a Saral vertical axis high-shear mixer granulator fitted with a 3.5-L bowl (Saral Engineering Company, Vapi, India). A model 7521-001 Cole-Palmer peristaltic pump (Cole-Palmer Instruments Co., Barrington, Illinois, USA) consisting of a spray gun fitted with a pressure gauge was used for the addition of binder solution. The granules were dried using a Memmert dry-heat oven (Memmert GmbH Co, Schwabach, Germany), and tablet compression was performed on a Manesty B3B rotary tablet press (Manesty, Speke, Liverpool, England) tooled with six 12-mm, flat-faced punches. All materials were sieved using wire cloth sieves conforming to DIN 4188 standards.

\section{Method of Manufacture}

RTD, MTZ, and MCC were weighed according to the formulation summarized in Table 1, passed through a screen of $850-\mu \mathrm{m}$ aperture size, and blended with half of the CCS at an impeller speed of $100 \mathrm{rpm}$. The resultant blend was granulated using a $12.5 \% \mathrm{~m} / \mathrm{v}$ solution of PVP-K30 at impeller and chopper speeds of 100 and $1000 \mathrm{rpm}$, respectively. The granulation was mixed for a further minute after completion of wetting of the powder mass, and the power consumption of the equipment was used to establish the endpoint of granulation prior to tray drying in an oven set at $50 \pm 0.5^{\circ} \mathrm{C}$ for $24 \mathrm{~h}$. The dried granules were passed through a $350-\mu \mathrm{m}$ screen and lubricated for an additional 3 min with previously screened colloidal silicone dioxide, magnesium stearate, and the remaining CCS (10 g). The lubricated granules were compressed into 500-mg tablets at a press speed of $25 \mathrm{rpm}$ to a target hardness of between 80 and $120 \mathrm{~N}$.

\section{Uniformity of Weight}

The individual weights of ten randomly selected tablets were determined using a Model AG 135 top-loading electronic balance (Mettler Instruments, Zurich, Switzerland) with a sensitivity of $0.1 \mathrm{mg}$, and the average weight was calculated.

\section{Friability}

The friability of 20 randomly selected tablets was determined using a Model TA3R friabilator (Erweka GmbH, Heusenstamm, Germany). The compressed tablets were dedusted and weighed using a top-loading balance (Model PM 4600, Mettler Instruments, Zurich, Switzerland). The tablets were allowed to tumble at $25 \mathrm{rpm}$ for $4 \mathrm{~min}$ (100 drops), removed, dedusted, reweighed, and the friability established.

\section{Disintegration}

Six tablets were selected randomly for the determination of disintegration time using a Model ZT 61 tablet disintegration apparatus (Erweka GmbH, Heusenstamm, Germany). Each tablet was placed into a cylinder of the basket rack and covered with a disc. The basket was set to oscillate vertically inside a beaker containing $700 \mathrm{~mL}$ of distilled water maintained at $37 \pm 0.2^{\circ} \mathrm{C}$ at a speed of 30 cycles/min. The time for disintegration of each tablet was recorded automatically on completion of the test.

\section{Experimental Design}

The evaluation of the main effects, interaction, and quadratic effects of the input and response variables was performed using a Box-Behnken statistical screening design that had three center points. The mathematical relationship between the input and output variables was generated using Design-Expert 8.0.4 software (Stat-Ease, Inc., Minneapolis, Minnesota, USA). The independent variable levels were established based on the Level 2 component and composition changes that have been described in SUPAC-IR (1), and these were studied at three levels (i.e., high, medium, and low). The levels and respective combination sequence of independent variables generated using the Box-Behnken approach is described in Table 2.

\section{Statistical Analysis}

The significance of the model and the model terms that were generated were analyzed using analysis of variance (ANOVA) type three (partial sum of squares) at a $5 \%$ level of significance using the statistical package Design Expert 8.0.4 (Stat-Ease, Inc., Minneapolis, Minnesota, USA). The predicted residual error sum of squares (PRESS) was used to assess which of the input factors had a significant impact on the measured response(s). A backward elimination procedure was used to fit data into the 
Table 2. Levels and Combination Sequence of Independent Variables and Respective Codes Used for the Box-Behnken Design

\begin{tabular}{|c|c|c|c|}
\hline \multirow[b]{2}{*}{ Independent Variable } & \multicolumn{3}{|c|}{ Levels Used, Actual (Coded) } \\
\hline & Low (-1) & Medium (0) & High (+1) \\
\hline$x_{1}=\operatorname{CCS}(\% \mathrm{w} / \mathrm{w})$ & 3 & 4 & 5 \\
\hline$x_{2}=$ PVP $-K 30(\% \mathrm{w} / \mathrm{w})$ & 0 & 1 & 2 \\
\hline \multirow[t]{2}{*}{$\begin{array}{l}x_{3}=\text { Magnesium } \\
\text { stearate }(\% \mathrm{w} / w)\end{array}$} & 0.5 & 1 & 1.5 \\
\hline & \multicolumn{3}{|c|}{ Independent Variables (\%w/w) } \\
\hline Batch Number* & $x_{1}$ & $x_{2}$ & $x_{3}$ \\
\hline RM 001 & 3.0 & 0.0 & 1.0 \\
\hline RM 002 & 5.0 & 0.0 & 1.0 \\
\hline RM 003 & 3.0 & 2.0 & 1.0 \\
\hline RM 004 & 5.0 & 2.0 & 1.0 \\
\hline RM 005 & 3.0 & 1.0 & 0.5 \\
\hline RM 006 & 5.0 & 1.0 & 0.5 \\
\hline RM 007 & 3.0 & 1.0 & 1.5 \\
\hline RM 008 & 5.0 & 1.0 & 1.5 \\
\hline RM 019 & 4.0 & 0.0 & 0.5 \\
\hline RM 010 & 4.0 & 2.0 & 0.5 \\
\hline RM 011 & 4.0 & 0.0 & 1.5 \\
\hline RM 012 & 4.0 & 2.0 & 1.5 \\
\hline RM 013 & 4.0 & 1.0 & 1.0 \\
\hline RM 014ロ & 4.0 & 1.0 & 1.0 \\
\hline RM 015 $\square$ & 4.0 & 1.0 & 1.0 \\
\hline
\end{tabular}

*Indicates arrangement by standard order

ulndicates center-point formulations

different predictor equations, and the negligible effect of omission of nonsignificant factors was confirmed following comparison of the predicted and adjusted $R^{2}$ values of the full and reduced model. Furthermore, predicted versus actual data diagnostic plots were used to determine the goodness of fit of the proposed model to the experimental data.

\section{In Vitro Release of Metronidazole and Ranitidine}

The FDA suggests that a drug product is considered rapidly dissolving when no less than $85 \%$ of the label claim dissolves within 30 min in solutions of $\mathrm{pH}$ values that represent the gastrointestinal tract $\mathrm{pH}$ range (15). MTZ and RTD are classified as rapidly dissolving drugs according to $\mathrm{BCS}(15,17,18)$. The high solubilities of MTZ and RTD prevent the use of 30 min as a discriminatory sampling time point to determine the effect of formulation variables on drug release in any critical manner; therefore, $10 \mathrm{~min}$ was adopted as the discriminatory
Table 3. Weight Uniformity, Disintegration Time, and Friability Values for the Tablets

\begin{tabular}{lc}
\hline Parameter & Value \\
\hline Weight uniformity $(\mathrm{mg})$ & $494.19 \pm 3.15-509.91 \pm 3.21$ \\
\hline Disintegration time $(\mathrm{min})$ & $3.58 \pm 0.49-11.41 \pm 0.21$ \\
\hline Friability (\%) & $<0.30$ \\
\hline
\end{tabular}

sampling time point. In vitro release studies of the FDC were performed using USP Apparatus 2 (Hanson Research SR 8 PLUS, Chartsworth, California, USA) fitted with an Autoplus Multifill and Maximizer Syringe Fraction Collector. Six tablets were dropped into the dissolution vessels each containing $900 \mathrm{~mL}$ of degassed $0.1 \mathrm{M} \mathrm{HCl}(\mathrm{pH}$ 1.2). The paddles were set to rotate at $75 \mathrm{rpm}$ since this speed represented a suitable compromise between the 50 and $100 \mathrm{rpm}$ specifications described in the USP for RTD and $M T Z$, respectively. The temperature of the dissolution medium was maintained at $37 \pm 0.5^{\circ} \mathrm{C}$, and 5 - $\mathrm{mL}$ aliquots were collected for analysis at intervals of $10,20,30$, and 45 min with replacement of an equal volume of fresh dissolution medium after removal of each sample. Correction of sample volume was performed prior to analysis of the dissolution data.

\section{Analytical Method}

MTZ and RTD were analyzed using a reversed-phase HPLC method that had previously been validated (19). Separation was achieved using a Nova-Pak $C_{18} 3.9 \times 150$ mm HPLC cartridge column (Waters Corporation, Milford, Massachusetts, USA) and a mobile phase that comprised $9 \%(\mathrm{v} / \mathrm{v})$ acetonitrile in phosphate buffer solution $(50 \mathrm{mM})$ containing $0.1 \%(\mathrm{v} / \mathrm{v})$ triethylamine at a $\mathrm{pH}$ of 6.7. A modular HPLC system (Thermo Separation Products, San Jose, California, USA) with a UV detector (Linear Instrument Co., California, USA) was used. The flow rate was set at 2 $\mathrm{mL} / \mathrm{min}$, and detection was achieved at $317 \mathrm{~nm}$.

\section{RESULTS AND DISCUSSION}

\section{Physicochemical Properties of Tablets}

The weight uniformity, disintegration time $\left(D_{t}\right)$, and friability of the compressed tablets complied with compendial specifications (Table 3).

\section{In Vitro Release}

For ease of determination of the quantitative and qualitative effects of input factors on drug release, the $Q_{10}$ values for MTZ and RTD from formulations manufactured with different levels of input factors were compared with those from formulations manufactured with intermediate levels of excipient composition (i.e., the center formulations). The notation used to depict the formulations uses the ratios of CCS:PVP-K30:magnesium stearate, and this convention has been adopted for the following discussion. By way of example, the center-point formulations are denoted 40:10:10. 


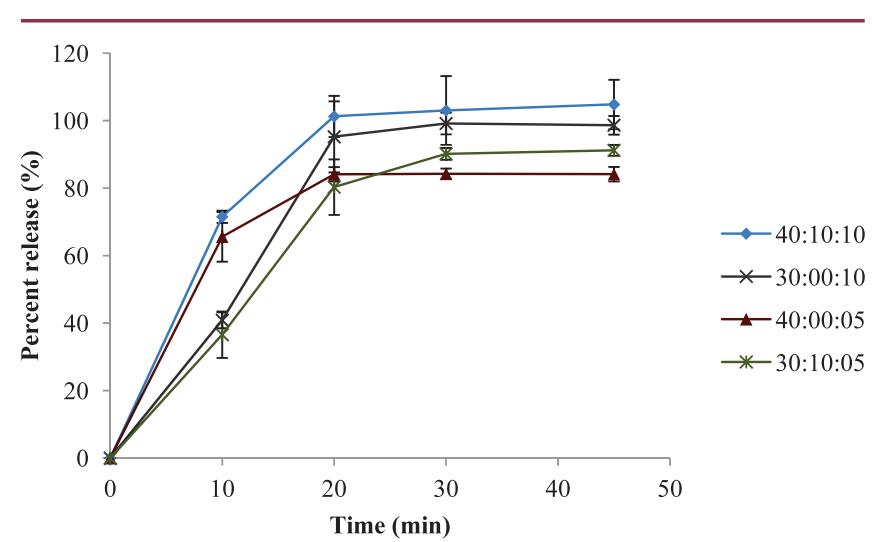

Figure 1. In vitro release profiles of MTZ for formulations containing CCS, PVP-K30, and magnesium stearate at low levels compared with the center formulation.

\section{In Vitro Release of Metronidazole from Formulations with Low Levels of Input Factors}

The effect of low levels of CCS, PVP-K30, and magnesium stearate in different ratios on the dissolution of MTZ is depicted in Figure 1. The center formulation exhibited $71 \%$ drug release within 10 min for MTZ, and $100 \%$ was released within 20 min of the commencement of dissolution testing. A reduction in the levels of CCS and the exclusion of PVP-K30 in formulation 30:00:10 resulted in a significant effect on the $Q_{10}$ value for MTZ (i.e., $40 \%$ released); however, the total amount of MTZ released was unaffected. Formulation 40:00:05 exhibited a relatively minor decrease in $Q_{10}$ for MTZ (i.e., 65\%) when PVP-K30 was excluded and magnesium stearate was included at low levels; however, the overall amount of MTZ released was unaffected with $84 \% \mathrm{MTZ}$ released after $45 \mathrm{~min}$. A reduction in CCS and magnesium stearate to minimum levels (formulation 30:10:05) resulted in a dramatic reduction in $Q_{10}$ for $M T Z$ to $36 \%$; however, approximately $91 \%$ MTZ was released within $30 \mathrm{~min}$. The $Q_{10}$ for MTZ is dependent on the content of CCS. Furthermore, the exclusion of PVP-K30 and the use of low levels of magnesium stearate have a relatively minor effect on the release of MTZ as evidenced by the changes observed for $Q_{10}$.

\section{In Vitro Release of Ranitidine from Formulations with Low Levels of Input Factors}

The effect of low levels of CCS, PVP-K30, and magnesium stearate in different ratios on the dissolution of RTD is depicted in Figure 2. The release of RTD from the center formulation exhibited a burst release similar to that of MTZ with a $Q_{10}$ value of $67 \%$ and $89 \%$ RTD released within $20 \mathrm{~min}$. The exclusion of PVP-K30 and the reduction of CCS to a lower level in formulation 30:00:10 resulted in a decrease in $Q_{10}$ for RTD to $50 \%$. However, the total drug released was unaffected since $92 \%$ RTD was released within $20 \mathrm{~min}$. The reduction of magnesium stearate to $0.5 \% \mathrm{w} / \mathrm{w}$ and the exclusion of PVP-K30 in formulation 40:00:05 had a relatively minor effect on the $Q_{10}$ for RTD compared with that observed for the center formulation. Formulation 40:00:05 exhibited a

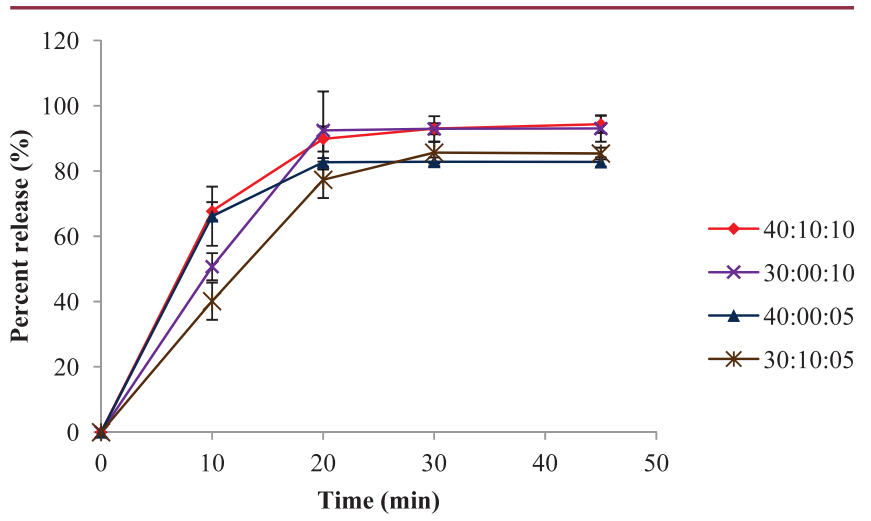

Figure 2. In vitro release profiles of RTD for formulations containing CCS, PVP-K30, and magnesium stearate at low levels compared with the center formulation.

$Q_{10}$ of $66 \%$ for RTD, and $83 \%$ of RTD was released within 20 $\min$. The reduction of both CCS and magnesium stearate to low levels (i.e., 3 and $0.5 \% \mathrm{w} / \mathrm{w}$, respectively) in formulation 30:10:05 resulted in a $Q_{10}$ of $40 \%$ for RTD and an almost two-fold increase in the extent of drug released to a value of $77 \%$ after 20 min compared with the $Q_{10}$, and $85 \%$ release was achieved within $30 \mathrm{~min}$. Therefore, it is clear that the dissolution of RTD also depends on the amount of CCS in the formulation. Furthermore, magnesium stearate and PVP-K30 exhibited relatively minor effects on the dissolution of RTD compared with that observed for the center formulation. However, at low levels of CCS (i.e., 3\% w/w in formulations 30:00:10 and 30:10:05), the dissolution profiles suggest that PVP-K30 has a greater retardation effect on the dissolution of RTD compared with the effect observed for magnesium stearate when using this $Q_{10}$ approach to evaluate the data.

\section{In Vitro Release of Metronidazole from Formulations with High Levels of Input Factors}

The effects of high levels of CCS, PVP-K30, and magnesium stearate on the release of MTZ from the tablets are depicted in Figure 3. Formulation 50:20:10, in which high concentrations of CCS and PVP-K30 were used, exhibited a burst release for MTZ with a resultant $Q_{10}$ of $82 \%$ and $97 \%$ of MTZ released after 20 min. High concentrations of CCS and magnesium stearate (50:10:15) had a relatively minor impact on the $Q_{10}$ for MTZ compared with that observed for the center formulation. Increasing the amount of PVP-K30 and magnesium stearate to $2 \%$ and $1.5 \% \mathrm{w} / \mathrm{w}$, respectively, as for formulation $40: 20: 15$, resulted in a $46 \%$ decrease in $Q_{10}$ for MTZ where $71 \%$ was observed for the center formulation. It is apparent that the $Q_{10}$ for MTZ is dependent on the amount of CCS used in the formulation. Moreover, the effects of PVP-K30 and magnesium stearate on the $Q_{10}$ of MTZ are also dependent on the CCS content. At high concentrations of CCS (i.e., $5 \%$ $\mathrm{w} / \mathrm{w}$ per tablet), variations in the amounts of PVP-K30 (i.e., formulation 50:20:10) and magnesium stearate (i.e., 


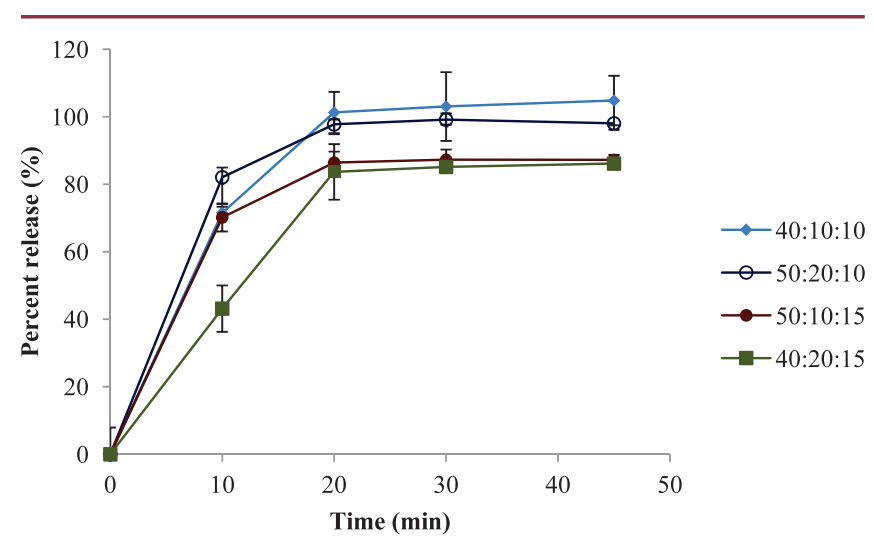

Figure 3. In vitro release profile of MTZ for formulations containing CCS, PVP-K30, and magnesium stearate at high levels compared with the center formulation.

formulation 50:10:15) exert a relatively minor effect on the $Q_{10}$ of MTZ. However, when the content of CCS is held constant at 4\% w/w as in formulation 40:20:15, an increase in PVP-K30 and magnesium stearate content results in a significant decrease in the $Q_{10}$ value for MTZ. The effect on dissolution of varying the amount of binder at constant levels of disintegrant has been reported (20). The decrease in the value for $Q_{10}$ observed following an increase in the content of PVP-K30 and magnesium stearate might be due to a combination of factors (e.g., formation of viscous or hydrophobic films, respectively). The inclusion of a high concentration of binder in a formulation may result in the formation of a viscous film on the surface of the tablet with a subsequent retardation of the dissolution of the API (21).

\section{In Vitro Release of Ranitidine from Formulations with High Levels of Input Factors}

The effects of high levels of CCS, PVP-K30, and magnesium stearate on the release of RTD are depicted in Figure 4. The in vitro dissolution profile of formulation 50:20:10, which was manufactured using high amounts of CCS and PVP-K30 (i.e., $5 \%$ and 2\% w/w per tablet, respectively) shows that a $Q_{10}$ value of $62 \%$ was obtained for RTD. The total RTD released (86\%) was observed within 20 min. A similar profile was also observed for formulation 50:10:15 in which an increase in the content of CCS and magnesium stearate resulted in a $Q_{10}$ of $72 \%$ and $90 \%$ RTD released within $20 \mathrm{~min}$. It is apparent that intermediate levels of PVP-K30 (i.e., 1\% w/w per tablet) and high levels of magnesium stearate and CCS facilitate the release of RTD. This observation indicates a possible synergistic interaction between CCS and magnesium stearate, which promotes the release of RTD.

In contrast, an increase in the content of magnesium stearate and PVP-K30 and the use of a constant level of CCS (i.e., formulation 40:20:15) results in the opposite effect. The $Q_{10}$ for RTD was reduced to $40 \%$ compared with that of $67 \%$ observed for the center formulation. The low $Q_{10}$ of $40 \%$ may be attributed to the high content of

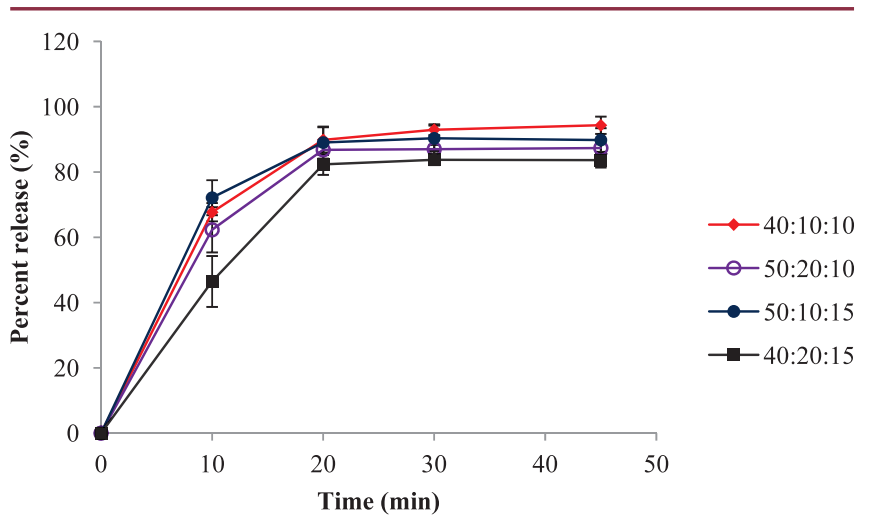

Figure 4. In vitro release profiles of RTD for formulations containing CCS, PVP-K30, and magnesium stearate at high levels compared with the center formulation.

PVP-K30 and magnesium stearate $(21,22)$. It is apparent that the overall $Q_{10}$ values for formulations that contain high and low amounts of CCS are similar, which suggests that CCS has a significant impact on the rate of drug release from these dosage forms. Further, the impact of PVP-K30 and magnesium stearate on drug release is dependent on the content of CCS, which validates the fact that CCS is an important formulation factor that determines the rate of drug release. However, the relationship between the factors and their levels and impact on the rate of drug release is complex and involves some degree of interaction, as shown for formulation 50:10:15 depicted in Figure 4. Therefore, RSM was used to investigate and explore the nature of this relationship and its potential impact on the release of MTZ and RTD.

\section{Response Surface Modeling}

The significance of the model terms and a model fit comparison for percent drug release in $10 \mathrm{~min}\left(Q_{10}\right)$ are summarized in Table 4. The resultant polynomial equations used for the investigation of the effect of input factors on $Q_{10}$ values for MTZ and RTD are described in eqs 1 and 2 , and the predicted versus actual diagnostic plots for the response variable $Q_{10}$ of both compounds are shown in Figure 5.

$$
\begin{aligned}
\boldsymbol{y}_{1}= & 72+22.94 x_{1}-5.98 x_{2}-3.88 x_{3}- \\
& 9.72 x_{1}^{2}-4.76 x_{2}^{2}-10.83 x_{3}^{2} \\
y_{2}= & 65.16+14.78 x_{1}-7.64 x_{2}-1.17 x_{3}+ \\
& 5.16 x_{1} x_{3}-8.65 x_{1}^{2}-6.19 x_{3}^{2}
\end{aligned}
$$

Evaluation of eq 1 reveals that CCS has a synergistic effect on the release of MTZ, whereas PVP-K30 and magnesium stearate have the opposite effect. These results are supported by the in vitro release profiles depicted in Figures 1 and 2. Similar results were also observed following an investigation into the effect of disintegrant, filler ratio, and lubricant levels on the in vitro release of propranolol hydrochloride (13). 
Table 4. Significant Model Terms and Model Fit Comparison of In Vitro Release of MTZ and RTD

\begin{tabular}{|c|c|c|c|c|}
\hline \multirow[b]{2}{*}{ Factor } & \multicolumn{2}{|c|}{ RTD } & \multicolumn{2}{|c|}{ MTZ } \\
\hline & $p$ value & Significance & $p$ value & Significance \\
\hline$x_{1}-\operatorname{CCS}$ & $<0.0001$ & Significant & $<0.0001$ & Significant \\
\hline$x_{2}-$ PVP-K30 & 0.0002 & Significant & 0.0073 & Significant \\
\hline $\begin{array}{l}x_{3} \text {-Magnesium } \\
\text { stearate }\end{array}$ & 0.3381 & $\begin{array}{c}\text { Not } \\
\text { significant }\end{array}$ & 0.0491 & Significant \\
\hline$x_{1} x_{2}$ & 0.7195 & $\begin{array}{c}\text { Not } \\
\text { significant }\end{array}$ & 0.1579 & $\begin{array}{c}\text { Not } \\
\text { significant }\end{array}$ \\
\hline$x_{1} x_{3}$ & 0.0131 & Significant & 0.5014 & $\begin{array}{c}\text { Not } \\
\text { significant }\end{array}$ \\
\hline$x_{2} x_{3}$ & 0.3020 & $\begin{array}{c}\text { Not } \\
\text { significant }\end{array}$ & 0.3271 & $\begin{array}{c}\text { Not } \\
\text { significant }\end{array}$ \\
\hline$x_{1}^{2}$ & 0.0009 & Significant & 0.0043 & Significant \\
\hline$x_{2}^{2}$ & 0.4609 & $\begin{array}{c}\text { Not } \\
\text { significant }\end{array}$ & 0.0894 & $\begin{array}{c}\text { Not } \\
\text { significant }\end{array}$ \\
\hline \multirow[t]{2}{*}{$x_{3}^{2}$} & 0.0063 & Significant & 0.0023 & Significant \\
\hline & \multicolumn{4}{|c|}{ PRESS } \\
\hline Model & \multicolumn{2}{|c|}{ RTD } & \multicolumn{2}{|c|}{ MTZ } \\
\hline Linear & \multicolumn{2}{|c|}{1000.83} & \multicolumn{2}{|c|}{1463.31} \\
\hline Quadratic & \multicolumn{2}{|c|}{367.24} & \multicolumn{2}{|c|}{682.17} \\
\hline
\end{tabular}

Similarly, CCS has a synergistic effect on the release of RTD, whereas PVP-K30 and magnesium stearate have an antagonistic effect as described in Equation 2. Furthermore, CCS and magnesium stearate exhibit a synergistic interaction on the release of RTD as can be seen from the positive value of the coefficient for this factor.

The impact of independent factors (i.e., CCS, PVP-K30, and magnesium stearate) at different levels on the in vitro release rate of MTZ and RTD is shown on contour and three-dimensional response surface plots in Figures 6-8 and $9-11$, respectively.

The three-dimensional response surface plot in Figure 6 depicts a curvilinear relationship between the factors and the response. The highest $Q_{10}$ for $M T Z \geq 80 \%$ is achieved when the levels of CCS are high and when those for PVP$\mathrm{K} 30$ are between a low and intermediate level. In addition, $\geq 80 \% \mathrm{MTZ}$ is released when the amounts of CCS and PVP-K30 per tablet are $>21 \mathrm{mg}$ and $<8 \mathrm{mg}$, respectively. It is evident that the antagonistic effect of PVP-K30 in the attainment of $Q_{10} \geq 80 \%$ for MTZ is achieved when the content of PVP-K30 per tablet is $>8 \mathrm{mg}$, at which point an increase in the content of CCS to maximum levels of 25 mg per tablet resulted in $<80 \%$ drug release. A similar antagonistic effect was observed when the amount of CCS per tablet was $15<x_{1}<21 \mathrm{mg}$ and a decrease in the $Q_{10}$ for MTZ was exhibited when the content of PVP-K30 was $>8 \mathrm{mg}$ per tablet. It can be concluded that a $Q_{10} \geq 80 \%$

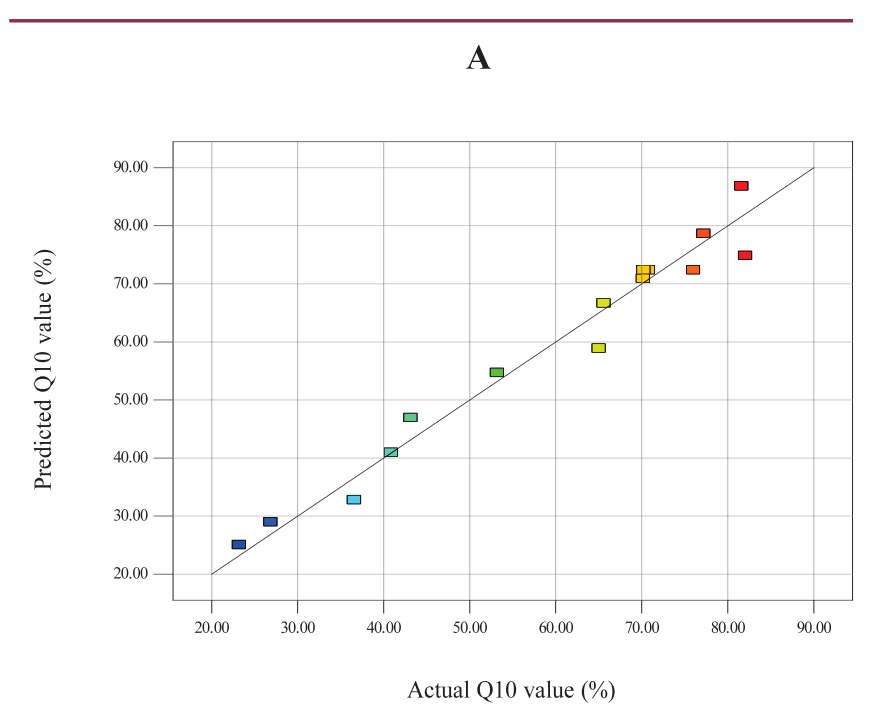

B

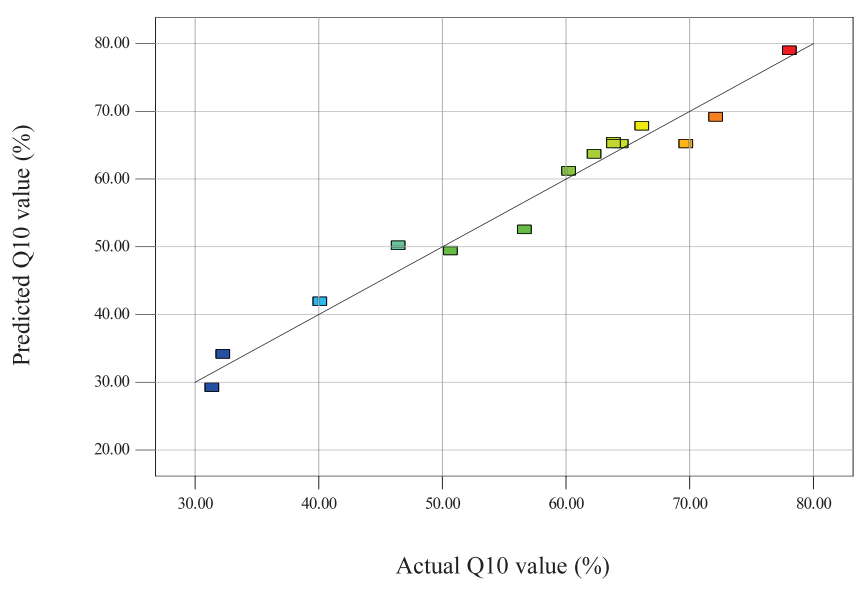

Figure 5. Predicted versus actual diagnostic plots for $Q_{10}$ values of (A) MTZ and (B) RTD.

for MTZ may be achieved when the amount of CCS and PVP-K30 per tablet is $>23 \mathrm{mg}$ and $<6 \mathrm{mg}$, respectively, and when an intermediate level of magnesium stearate is included in the formulation.

In general, the impact of magnesium stearate and PVP-K30 on the $Q_{10}$ value for MTZ is minor in comparison with the effect of CCS, as shown in Figure 7.It is clear that both factors have an antagonistic effect on drug release, and an increase in either factor results in a decrease in the value of $Q_{10}$ for MTZ. This effect is apparent when the amount of PVP-K30 is $>6 \mathrm{mg}$ per tablet and may in part be attributed to the highly hydrophilic nature of MTZ, which would require high concentrations of PVP-K30 to retard drug release by creating a viscous film on the tablet surface. A $Q_{10}$ value $\geq 70 \%$ for MTZ is achieved when the content of magnesium stearate per tablet $\left(x_{3}\right)$ is between 2.5 and $6.5 \mathrm{mg}$ and that of PVP-K30 is less than $6 \mathrm{mg}$ with CCS at an intermediate level of $20 \mathrm{mg}$ per tablet. 


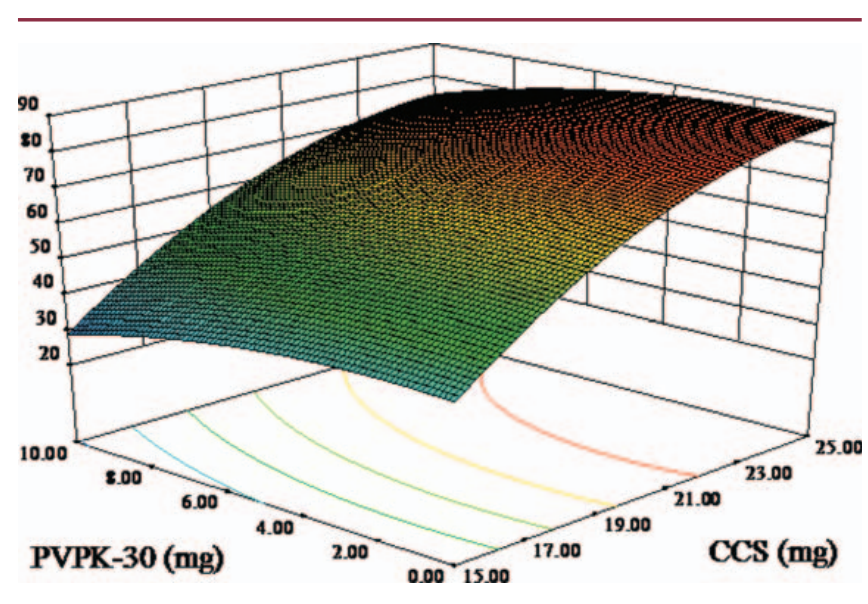

Figure 6. Three-dimensional response surface plot showing the impact of CCS and PVP-K30 on the $Q_{10}$ value for MTZ at intermediate levels of magnesium stearate.

Magnesium stearate exhibited an antagonistic effect, similar to the effects observed with PVP-K30 on the dissolution of MTZ as shown in the contour plot in Figure 8. However, this effect is marked when the content of magnesium stearate per tablet is $>5.5 \mathrm{mg}$. These results are supported by the in vitro release profile of formulation 40:20:15 depicted in Figure 3. The decrease in drug release observed at concentrations of magnesium stearate $>5 \%$ $\mathrm{w} / \mathrm{w}$ may be attributed to the hydrophobic nature of magnesium stearate that retards dissolution of the hydrophilic MTZ.

It is also evident that an increase in the levels of CCS resulted in an increase in drug release; however, a high value for $Q_{10} \geq 80 \%$ for MTZ was observed when the content of CCS and magnesium stearate per tablet was $>22 \mathrm{mg}$ and $<6.5 \mathrm{mg}$, respectively, as shown in Figure 8 .

The three-dimensional response surface plot for the $Q_{10}$ value for RTD is shown in Figure 9. It is curvilinear for the

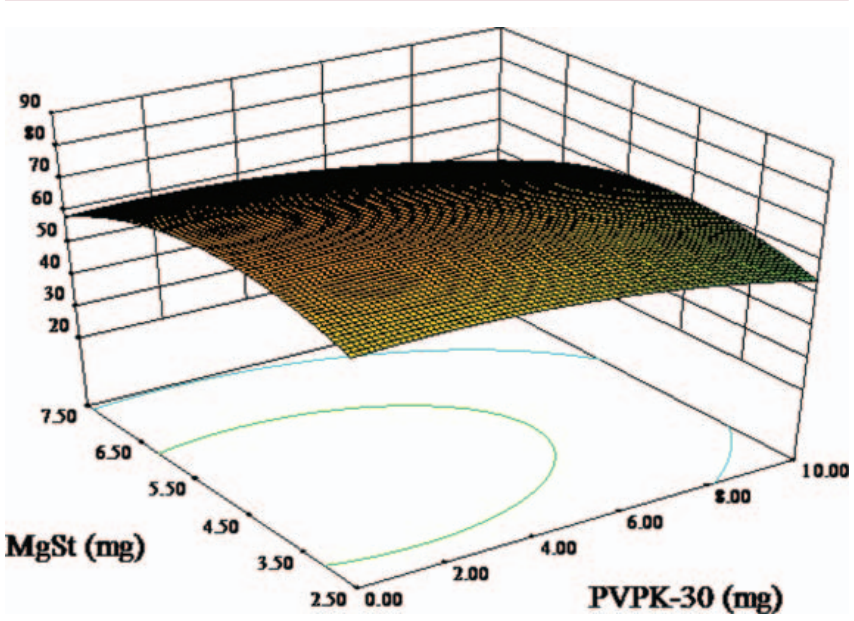

Figure 7. Three-dimensional response surface plot depicting the impact of magnesium stearate and PVP-K30 on the $Q_{10}$ value for MTZ at intermediate levels of CCS.

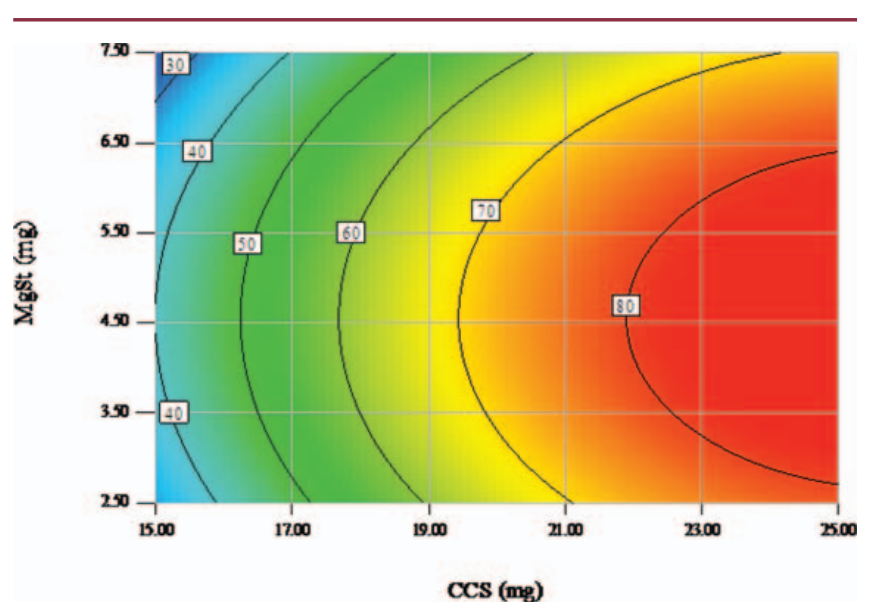

Figure 8. Contour plot showing the impact of magnesium stearate and CCS levels on the $Q_{10}$ value for MTZ at intermediate levels of PVP-K30.

relationship of this parameter with the amount of CCS as an input factor, whereas a linear relationship is observed for the effect of PVP-K30 levels on this measurement. As for MTZ, CCS has a profound effect on the release of RTD. An increase in the amount of CCS in the formulation results in an increase in the value of $Q_{10}$ for RTD; for a decrease in the amount of CCS, the converse is true. In contrast, PVP-K30 appears to exert an antagonistic effect on the value for $Q_{10}$ for RTD, and the impact is greater when the amount of CCS per tablet is $<17 \mathrm{mg}$ per tablet. At low levels of CCS, the $D_{t}$ of the tablets is increased as a consequence of the low content of CCS (23). The increase in $D_{t}$ results in a lower value of $Q_{10}$ for RTD, since the total surface area necessary for dissolution is smaller and therefore the impact of PVP-K30 on $D_{t}$ is more apparent. The response surface plot depicted in Figure 9 shows that a high $Q_{10}$ value of $\geq 78$ is achieved when the amount of CCS is $>23 \mathrm{mg}$ per tablet, when PVP-K30 is omitted from

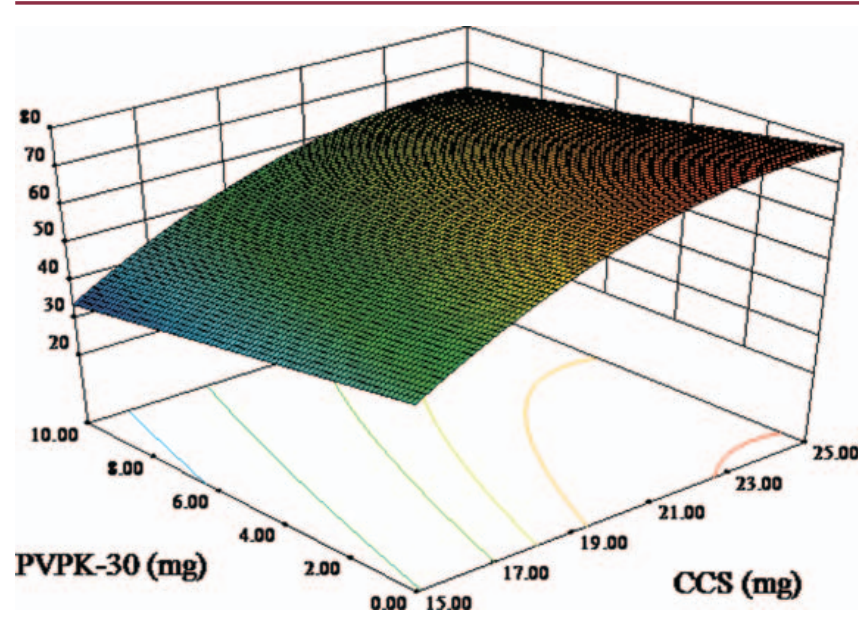

Figure 9. Three-dimensional response surface plot showing the impact of $P V P-K 30$ and CCS on the $Q_{10}$ value for RTD at intermediate levels of magnesium stearate. 


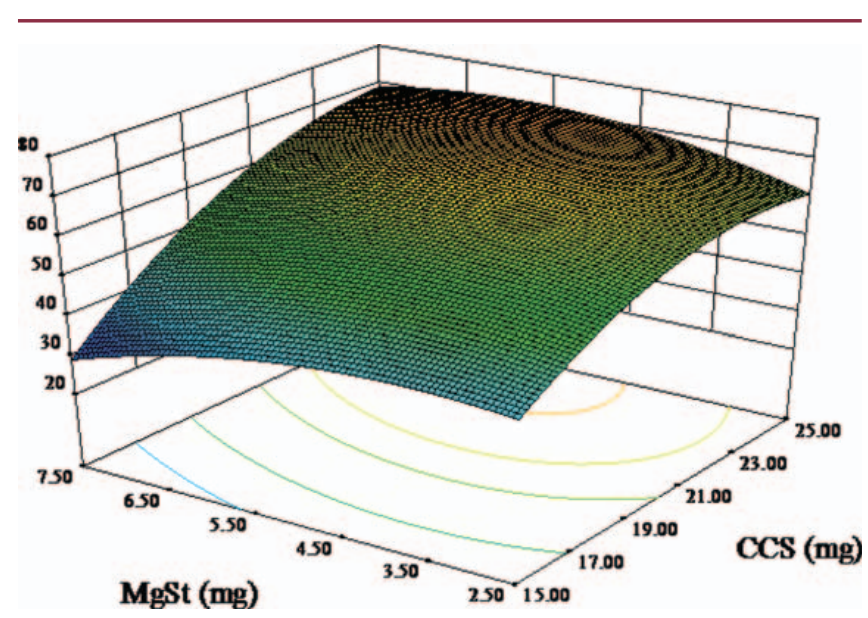

Figure 10. Three-dimensional response surface plot showing the impact of magnesium stearate and CCS on the value of $Q_{10}$ for RTD at intermediate levels of PVP-K30.

the tablet formulation and intermediate levels of Vmagnesium stearate are used.

In contrast to the effects of magnesium stearate on the value of $Q_{10}$ for $M T Z$, different results are observed when evaluating the $Q_{10}$ value for RTD. According to BCS, metronidazole is a Class 1 compound (i.e., highly soluble and highly permeable), and ranitidine is a Class 3 compound (i.e., highly soluble, poorly permeable). Consequently, the variability in physicochemical properties of these APIs is unlikely to have an impact on the $Q_{10}$ value since we are investigating the in vitro rate of solution, which is related to solubility and not the permeability of the molecules. A curvilinear relationship is exhibited for the effect of CCS and magnesium stearate on the value for $Q_{10}$ for RTD, and a region corresponding to maximum value for $Q_{10} \geq 70 \%$ is located between intermediate levels of magnesium stearate and high levels of CCS as depicted in Figure 10. These results suggest that a synergistic interaction exists between CCS and magnesium stearate at these levels (i.e., $\geq 23$ and $4.5 \leq x_{3}$ $\leq 6.5 \mathrm{mg}$ per tablet, respectively). Although this synergistic interaction has not been reported, the results are in agreement following an investigation of drug and excipient interactions with lubricants (24). Close inspection of the in vitro release profile for formulation 50:10:15 as shown in Figure 4 and evaluation of the resultant model equation for the parameter $y_{2}$ show that a synergistic interaction between CCS and magnesium stearate is evident.

The response surface plot depicted in Figure 11 reveals a linear antagonistic relationship between PVP-K30 and magnesium stearate on the value of $Q_{10}$ for RTD. It is clear that PVP-K30 exerts a greater effect on the $Q_{10}$ value than magnesium stearate, and consequently, the $Q_{10}$ value is not affected with an increase in the levels of magnesium stearate in the formulation. The effect of magnesium stearate is more apparent at low levels of PVP-K30

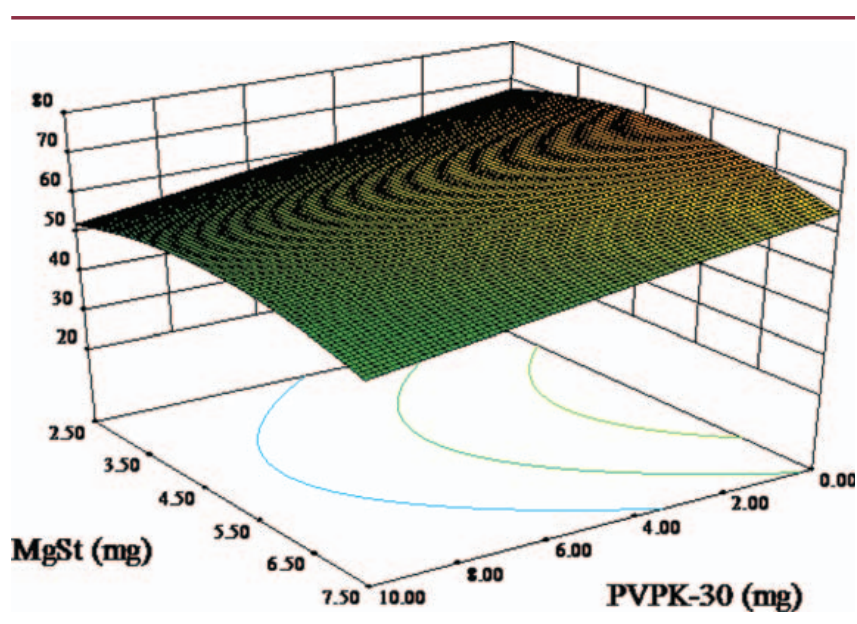

Figure 11. Three-dimensional response surface plot depicting the impact of $P V P-K 30$ and magnesium stearate on the value of $Q_{10}$ for $R T D$ at intermediate levels of CCS.

(i.e., $<2 \mathrm{mg}$ per tablet), as indicated by a $Q_{10}$ value of $<70 \%$ for RTD when the amount of lubricant per tablet is $\geq 6.5$ $\mathrm{mg}$ and where intermediate levels of CCS are used.

\section{CONCLUSIONS}

The use of RSM has facilitated the evaluation of the impact of SUPAC-IR Level 2 changes on the value of $Q_{10}$ for the fixed-dose combination tablets containing MTZ and RTD manufactured in these studies. Three-dimensional and contour-response surface plots clearly demonstrate the impact of the different levels of CCS, PVP-K30, and magnesium stearate on the in vitro release of MTZ and RTD. The response surface plots show that CCS is the most significant factor that affects the measured responses, and the effects of PVP-K30 and magnesium stearate are dependent on CCS levels. Moreover, magnesium stearate has a synergistic interaction with CCS, thereby promoting the in vitro release of RTD. Further investigation is warranted to determine the nature of interaction between CCS and magnesium stearate that promotes the dissolution of RTD. The use of this approach has permitted the identification of significant factors for the formulations studied in this research and provides a framework for further investigation to solving formulation challenges.

\section{ACKNOWLEDGMENTS}

The authors wish to acknowledge Research Committee of Rhodes University (RBW) for the financial assistance.

\section{REFERENCES}

1. Immediate Release Solid Oral Dosage Forms, Scale-Up and Postapproval Changes: Chemistry, Manufacturing, and Controls, In Vitro Dissolution Testing, and In Vivo Bioequivalence Documentation; Guidance for Industry; U.S. Department of Health and Human Services, Food and Drug Administration, Center for Drug Evaluation and Research (CDER), U.S. Government Printing Office: Washington, DC, 1995.

Dissolution Technologies I MAY 2012 
2. Khamanga, S. M.; Walker, R. B. The use of experimental design in the development of an HPLC-ECD method for the analysis of captopril. Talanta 2011,83 (3), 1037-1049.

3. Schwartz, J. B.; Flamholz, J. R.; Press, R. H. Computer optimization of pharmaceutical formulations I: General procedure.J. Pharm. Sci. 1973, 62 (7), 1165-1170.

4. Fonner, D. E., Jr.; Buck, J. R.; Banker, G. S. Mathematical optimization techniques in drug product design and process analysis. J. Pharm. Sci. 1970, 59 (11), 1587-1596.

5. Singh, B.; Kumar, R.; Ahuja, N. Optimizing drug delivery systems using systematic "design of experiments." Part I: fundamental aspects. Crit. Rev. Ther. Drug Carrier Syst. 2005, 22 (1), 27-105.

6. Aslan, N. Application of response surface methodology and central composite rotatable design for modeling and optimization of a multi-gravity separator for chromite concentration. Powder Technol. 2008, 185 (1), 80-86.

7. Lewis, G. A. Optimization Methods. In Encylopedia of Pharmaceutical Technology; Swarbrick, J., Ed.; Informa Healthcare: London, 2002; pp 1922-1937.

8. Adeyeye, M.C. Drug-Excipient Interaction Occurrences During Solid Dosage Form Development. In Preformulation in Solid Dosage Form Development; Adeyeye, M. C., Brittain, H. G., eds.; Informa Health Care: New York, 2008; pp 357-436.

9. Myers, R. H.; Montgomery, D. C.; Anderson-Cook, C.M. Response Surface Methodology: Process and Product Optimization Using Designed Experiments, 3rd ed.; John Wiley: Hoboken, NJ, 2009.

10. Bodea, A.; Leucuta, S. E. Optimization of Propranolol Hydrochloride Sustained-Release Pellets Using Box-Behnken Design and Desirability Function. Drug Dev. Ind. Pharm. 1998, 24 (2), 145-155.

11. Bodea, A.; Leucuta, S. E. Optimization of hydrophilic matrix tablets using a D-optimal design. Int. J. Pharm. 1997, 153 (2), 247-255.

12. Bodea, A.; Leucuta, S. E. Optimization of propranolol hydrochloride sustained release pellets using a factorial design. Int. J. Pharm. 1997, 154 (1), 49-57.

13. Eddington, N. D.; Ashraf, M.; Augsburger, L. L.; Leslie, J. L.; Fossler, M. J.; Lesko, L. J.; Shah, V. P.; Rekhi, G. S. Identification of formulation and manufacturing variables that influence in vitro dissolution and in vivo bioavailability of propranolol hydrochloride tablets. Pharm. Dev. Technol. 1998, 3 (4), 535-547.

14. Chaibva, F.; Burton, M.; Walker, R. Optimization of Salbutamol Sulfate Dissolution from Sustained Release Matrix Formulations Using an Artificial Neural Network. Pharmaceutics 2010, 2 (2), 182-198.

15. Waiver of In Vivo Bioavailability and Bioequivalence Studies for Immediate-Release Solid Oral Dosage Forms Based on a Biopharmaceutics Classification System; Guidance for Industry; U.S. Department of Health and Human Services, Food and Drug Administration, Center for Drug Evaluation and Research (CDER), U.S. Government Printing Office:Washington, DC, 2000.

16. Baren, K. I. Drugs of Acid Related Disorders. In South Africa Medicines Formulary, 8th ed.; Gibbon, C. J., Blockman, M., Eds.; Health and Medical Publishing Group of the South African Medical Association: Cape Town, 2008; pp 39-44.

17. Ahmad, M.; Pervaiz, K.; Murtaza, G.; Razman, M. Pharmacokinetic modelling of microencapsulated metronidazole. Acta Pharmacol. Sin. 2009, 44 (6), 674-679.

18. Blume, H. H.; Schug, B. S. The biopharmaceutics classification system (BCS): Class III drugs-better candidates for BA/BE waiver? Eur. J. Pharm. Sci. 1999, 9 (2), 117-121.

19. King'ori, D. L. The Development and Assessment of a Fixed Dose Combination Tablet of Ranitidine and Metronidazole. M.S. Thesis, Rhodes University, Grahamstown, South Africa, 2011; p 41.

20. Gupta, A.; Hunt, R.; Shah, R.; Sayeed, V.; Khan, M. Disintegration of highly soluble immediate release tablets: A surrogate for dissolution. AAPS PharmSciTech 2009, 10 (2), 495-499.

21. Parmar, J.; Rane, M. Tablet Formulation Design and Manufacture: Oral Immediate Release Application. Pharma Times 2009, 41 (4), 21-29.

22. Dilova, V.; Miteva, Z.; Arnaudova, P. Influence of lubricants on physicomechanical and kinetic parameters of tablets. Pharmacia 2006, 53 (1), 21-23.

23. Massimo, G.; Catellani, P. L.; Santi, P.; Bettini, R.; Vaona, G.; Bonfanti, A.; Maggi, L.; Colombo, P. Disintegration propensity of tablets evaluated by means of disintegrating force kinetics. Pharm. Dev. Technol. 2000, 5 (2), 163-169.

24. Wang, J.; Wen, H.; Desai, D. Lubrication in tablet formulations. Eur. J. Pharm. Biopharm. 2010, 75 (1), 1-15. 